\title{
Investigation on rolling sliding wear behavior of wheel steel by laser dispersed treatment
}

\author{
Zheng-yang $\mathrm{Li}^{\mathrm{a}, *}, \mathrm{Xu}$-hui Xing ${ }^{\mathrm{a}}$, Ming-jiang Yang ${ }^{\mathrm{a}}$, Bing Yang ${ }^{\mathrm{a}}$, \\ Zhi-yuan Ren ${ }^{a}$, Hua-qiang Lin $^{b}$ \\ a Institute of Mechanics, Chinese Academy of Sciences, Beijing 100190, PR China \\ ${ }^{\mathrm{b}}$ National Engineering Laboratory for System Integration of High-speed Train (South), CSR Qingdao Sifang Co., Ltd., Qingdao, Shandong 266111, PR China
}

\section{A R T I C L E I N F O}

\section{Article history:}

Received 27 November 2013

Accepted 7 December 2013

Available online 18 December 2013

\section{Keywords}

Rolling-sliding wear

Wheel/rail

Laser treatment

Steel

\begin{abstract}
A B S T R A C T
To improve wear resistance of wheel steels under rolling-sliding wear conditions, a novel technique named laser dispersed treatment (LDT) was used by forming a regular scattered hardening pattern distributed on the surface of wheel disc, which can avoid soft zones induced by tempering effect or cracks in the overlapped region. The wear rate of LDT wheel disc was evaluated by twin-disc wear testing machine in comparison with the untreated disc. The microstructure of LDT wheel disc and untreated disc was characterized by optical microscopy (OM), X-ray diffraction (XRD) and scanning electron microscopy (SEM). This study focuses on the relationship between the of microstructure and wear resistance of laser treated zone. It is shown that a higher wear resistance of laser treated zone is attributed to the strain strengthen of retained austenite during wearing and the compressive stress within laser treated zone. The laser dispersed treatment on wheel may be a useful technique to improve the wear resistance of wheel without harmful influence on rail.
\end{abstract}

(C) 2013 Elsevier B.V. All rights reserved.

\section{Introduction}

With a steady increase in loads and total traffic that railroads tracks have to carry, especially by high speed train and heavy load railway, wheel/rail is required to sustain service conditions more severe than those considered in their original design. An excessive wear of the wheel/rail results in reduced in wheel/rail life. To prolong lifetime of wheel/rail, many attempts based on wholly heat treatment of the materials, have been made to improve wear resistance of wheel/rail steels under rolling-sliding wear conditions [1-5]. As is well known, the failure of rolling-sliding wear is primarily determined by the surface properties of a material rather than by the bulk properties. With this respect, application of appropriate surface techniques offers feasible avenues for protection wheel/rail from wear damage. Among the surface techniques, laser surface modification is one of the promising due to its high cooling rates up to $10^{3}-10^{8} \mathrm{~K} / \mathrm{s}$ and yielding rapid resolidification [6]. In the last years, a series of laser surface modification technologies, such as laser hardening, laser melting, or laser cladding of rail/wheel have been developed for improving wear resistance of wheel/rail [7-10]. These laser surface modification technologies are mainly used to prepare the hard surface layers on ferrous materials via martensitic phase transformation or by

\footnotetext{
* Corresponding author. Tel.: +8610 82544258; fax: +8610 82544261 .

E-mail address: zyli@imech.ac.cn (Z.-y. Li).
}

adding alloy elements forming solution hardening layer. A major advantage of this process is the metallurgically bonded between the substrate and coatings free of debonding or spalling. Owing to the limited size of the laser beam, just a restricted space is dealt with using a single laser scanning, and thus multiple overlapping laser tracks is indispensable when a large area required to be treated $[11,12]$. However, multiple passes with overlapping laser tracks is harmful due to the formation of soft zones induced by tempering effect or cracks in the overlapped region [13]. Van Brussel et al. stated that the stress profile in multiple overlapping laser tracks was determined by laser processing and the materials being treated. The residual stress state in multiple laser tracks is of tensile character. Each new laid laser track exerts a tensile force upon the previous laser tracks, wherein the compressive stresses disappear [14,15].

Laser dispersed treatment (LDT) is one of the reasonable ways to address the above problem, which effectively avoids the overlapping of laser treated zone by creating isolated hardening, melted zones arranged in certain patterns on the component surface $[16,17]$. It is apparent that the dispersed, isolated hardening zones provide several unique advantages for improving wear performance of materials as compared to the conventional laser surface methods using multiple overlapping laser tracks [18]:

- Avoidance of hardness reduction of previously formed laser hardening zones subjected to the subsequent laser irradiation, since the resultant heat affected zones between adjacent laser hardening zones are devoid of overlap by adjusting spacing. 
- Reduction/elimination of stresses and distortions.

- Increase of the productivity due to only a fraction of the surface needed to be treated to achieve the same levels of wear performance.

Furthermore James et al. reported that compressive residual stress was obtained in the last pass of multiple overlapping laser remelted 4140 steel while tensile stress still existed in other overlapping laser tracks [19]. The result indicated that the martensite phase would be obtained by rapid self-cooling during the laser melting process for high or medium carbon steel given that no laser tracks reheating occurred.

In the current work, a pulse laser beam was performed to prepare a regular scattered melting/hardening spot pattern distributed on the surface of wheel steel. As is well known, the wear condition of tread and rim of wheel is far different. The tread works under the condition of rolling and sliding, it is expected small slip ratio and relatively high traction force at starting and braking. Thus the scattering distributed laser hardened pattern may not only improve the wear resistance of wheel tread, but enhance traction as well at starting and braking. This study focus on the wear rate of wheel steel and reveal the wear mechanism of laser treated zone and its regular scattered pattern.

\section{Experiments}

The wheel steel and rail steel used in this study were CL60 and U71Mn, respectively, which were typical wheel and rail material in China railway system. Table 1 depicts the chemical composition of rail and wheel steels used in the present study. Rolling and sliding wear experiments were conducted on an Amsler type wear testing machine. Two rollers with $45 \mathrm{~mm}$ diameter and $10 \mathrm{~mm}$ thickness with $5 \mathrm{~mm}$ contact strip were fabricated by wheel steel and rail steel, respectively. The rollers were mounted on two counterrotating geared axles. The lower disc was driver and the upper disc was driven. The driver (lower disc mounted) was controlled by a servo motor. An Eddy Current Brake (E.C.B.) was employed on the upper disc side, so that the driven disc was applied by a braking torque to control the slip ratio between the two rollers continuously. In this study only wheel disc was laser treated and the rail disc was untreated merely as a referential disc. Two series experiment were carried out, one was untreated wheel disc matching rail disc and the other was LDT wheel disc matching rail disc, i.e. CL60 against U71Mn and LDTCL60 against U71Mn. The surface of wheel discs was treated by using a pulse YAG laser set under an argon gas shield. The diameter of laser beam was $1 \mathrm{~mm}$ and the duration of laser pulse was $10 \mathrm{~ms}$. The pulse energy of a single laser was around $9 \mathrm{~J}$. The laser coverage ratio, which is the ratio of the area of LDT zone to cylindrical surface of the roller, is around $80 \%$. Before performing wear tests, both treated and untreated disc were surface ground to surface roughness $R a=0.8 \mu \mathrm{m}$. In this study the slip ratio is controlled around $0.5 \%$, while the traction coefficient is around 0.263 . The normal load is $1400 \mathrm{~N}$ loaded by screwing a spring. Then the maximum contact pressure, tractive force and the wear index $\left(\mathrm{T}_{\gamma} / \mathrm{A}\right)$ are $978 \mathrm{MPa}$, $368 \mathrm{~N}$ and $1.01 \mathrm{~N} / \mathrm{mm}^{2}$, respectively. Wear rate (microgram per meter rolled per square millimeter of contact area) were

Table 1

Chemical composition of rail and wheel steels.

\begin{tabular}{llllll}
\hline & C & Si & Mn & S & P \\
\hline Rail & $0.65-0.76$ & $0.15-0.35$ & $1.10-1.40$ & $\leq 0.03$ & $\leq 0.03$ \\
Wheel & $0.55-0.65$ & $0.17-0.37$ & $0.5-0.8$ & $\leq 0.03$ & $\leq 0.03$ \\
\hline
\end{tabular}

determined by measuring the weight loss of the rollers over a given period. The laser dispersed treatment and the untreated sample were prepared and examined by X-ray diffraction (XRD, Bruker D8 Advance with Cu Ko radiation), FEI Sirion 400NC scanning electron microscope (SEM) and optical microscope. The hardness was measured by MH-7 microhardness tester with a load of $200 \mathrm{~g}$ and loading time of $10 \mathrm{~s}$.

\section{Results}

\subsection{Laser processing and microstructure of laser treated zone}

According to the dimension of the roller and normal load as described in Section 2, the half with of contact strip $b$ is $0.182 \mathrm{~mm}$. To evaluate the effect of wear resistance of laser treated sample, several spots must be presented within the contact strip. After laser processing parameter optimization, we select a diameter of laser spot of $\sim 1 \mathrm{~mm}$ and the center spacing between two adjacent spots around 1-1.5 times spot diameter. Single laser spot should meet the following criteria: minimization of the porosity and cracking tendency and maximization of depth of the treated zone.

Fig. 1 shows the laser spots pattern covering the cylindrical surface of wheel disc without overlapping. The differences of dimension and distance between the spots result from the instability of laser beam operated in the pulsed mode.

Fig. 2 shows the cross-section of a single LDT spot. Apparently there are two different zones within the spot. The marked A region with some white areas is laser melted zone (LMZ), whereas the surrounded marked B region with gray contrast is identified as laser heated zone (LHZ). The marked C region is substrate.

Fig. 3 presents the XRD patterns obtained from LDT sample and untreated substrate. The untreated wheel disc consists of two phases, namely, the ferrite and a small amount of cementite, corresponding to its microstructure of pearlite. After laser melt treatment, the peaks intensity of ferrite is obviously reduced with broadening XRD peaks, while the peaks of retained austenite appear. The broadening peaks is due to the presence of refined $\alpha$-Fe grains even nanocrystalline phases, arising from rapid solidification of the melt zone [20]. Moreover, some weak cementite peaks are observed from the exposed substrate between the two adjacent laser zones. Therefore, the microstructure of laser treated zone is composed of martensite and austenite. Shariff et al. also suggested that the microstructure of laser treated pearlite steel was composed of martensite and austenite [21].

Fig. 4 shows hardness profiles of laser treated zone from surface to depth around $700 \mu \mathrm{m}$. The microhardness of LHZ and LMZ is in the range of $700-850 \mathrm{HV}$ and $480-580 \mathrm{HV}$, respectively.

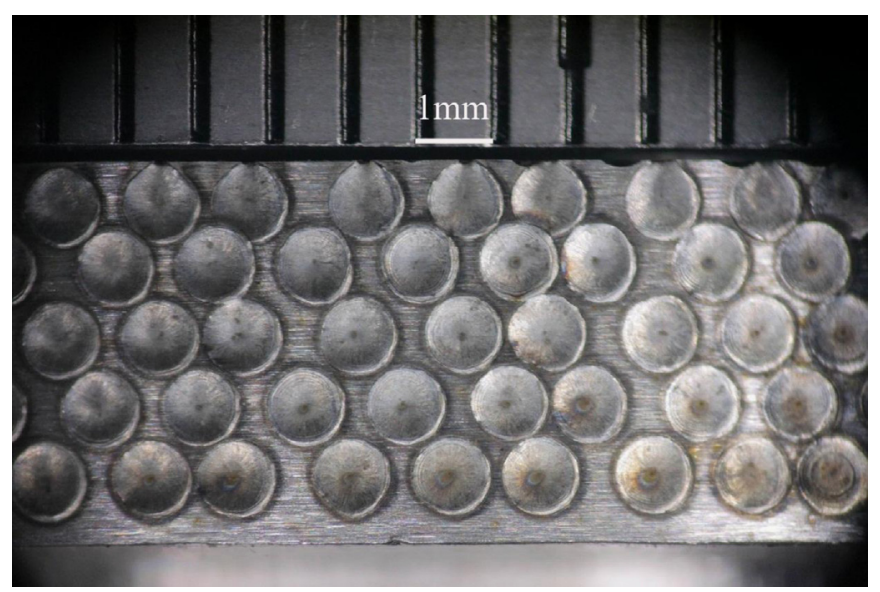

Fig. 1. LDT spots pattern before being grounded. 


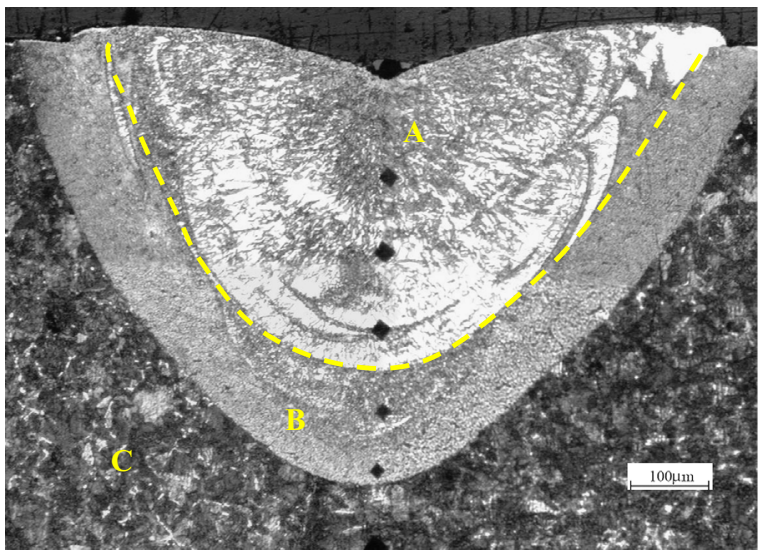

Fig. 2. Cross-section of single LDT zone before being grounded.

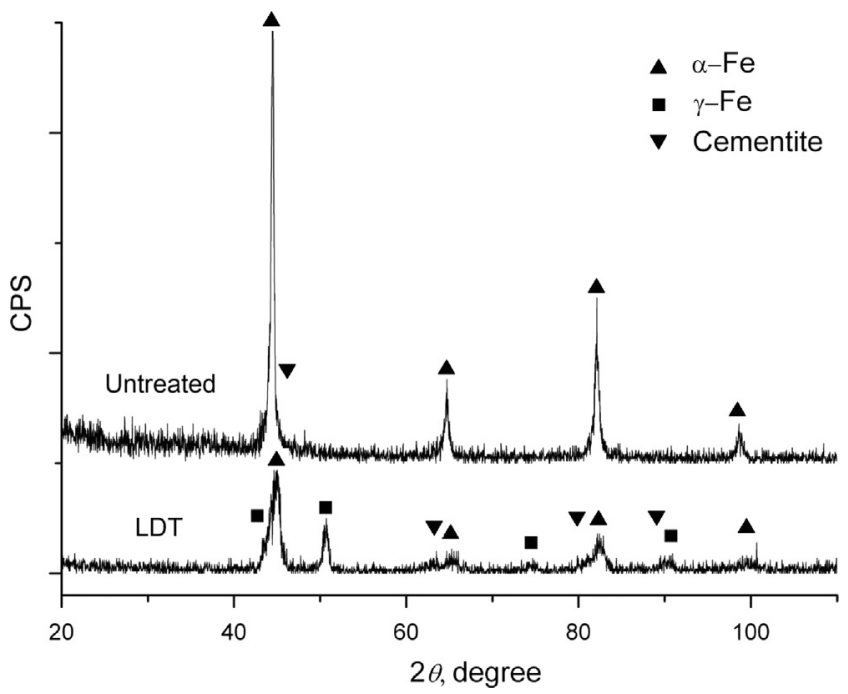

Fig. 3. XRD pattern of LDT wheel disc sample and the untreated wheel sample.

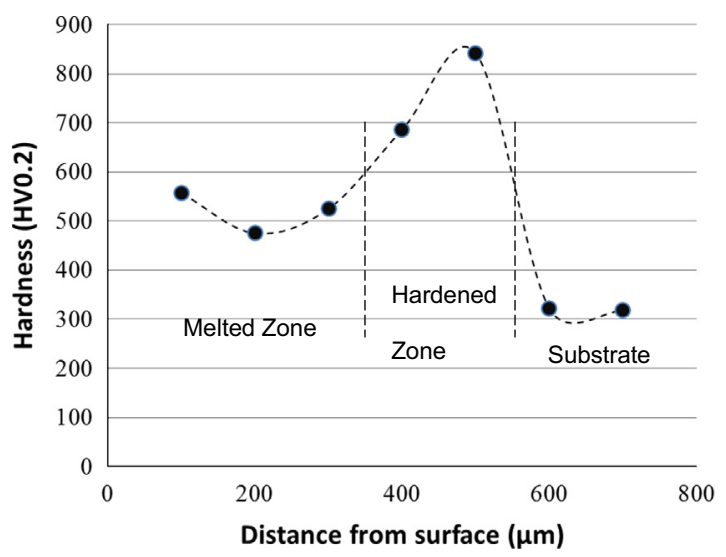

Fig. 4. Hardness profiles along depth in LDT zone.

Based on the discrepancy in microhardness of the two zones, it is inferred that some white area of LMZ with a lower hardness may contain more retained austenite, while LHZ with a higher hardness is mainly composed of the refined martensite free of austenite.

It is important to note that the amount of retained austenite plays a key role in controlling the wear resistance of laser treated zone. For the laser treated with a two phases structure, there is a critical amount of austenite; if the amount of retained austenite below this critical amount, the austenite shows the positive effect on wear resistance, otherwise, an adverse effect would be occurred
[22-24]. It is noted that the microstructure of laser treated zone was governed not only by chemical and phase composition of the treated materials, but also by laser processing, such as power density, scanning speed and diameter of laser beam. In this study, the power density of laser beam and laser irradiation duration used are around $10^{5} \mathrm{~W} / \mathrm{cm}^{2}$ and $10 \mathrm{~ms}$, respectively. Both the materials and the laser processing employed are similar to that of used in Ref. [16]. It was reported that the amount of retained austenite was around from $2.1 \%$ in hardened layer and 9.8\% in melt layer [20]. Therefore it is deduced that the amount of retained austenite in LDT zone is no more than $10 \%$ in this study.

\subsection{Wear test results}

Fig. 5 shows the wear rate variations of wheel and rail disc versus wear cycles. The wear rates of untreated wheel disc and rail disc samples are around 21 and $8 \mu \mathrm{g} / \mathrm{m} / \mathrm{mm}^{2}$, respectively. This indicates that these samples undergo the mild wear regime as reported by Lewis et al. [25,26] and Ekberg [27]. According to their results of twin disc tests, the wear process of railway wheel/rail steel could be classified into three kinds of regimes, i.e. mild, severe and catastrophe, and a traction coefficient below 0.3 was case of moderate surface friction. On the contrary, the wear rates of LDT wheel disc increases from around $14-20 \mu \mathrm{g} / \mathrm{m} / \mathrm{mm}^{2}$ with increasing the number of cycles, and after $3 \times 10^{5}$ cycles, its wear rate maintained around $22 \mu \mathrm{g} / \mathrm{m} / \mathrm{mm}^{2}$. Because the of laser treated zone is something like strawberry (Fig. 2), the surface area of a laser spot would gradually decrease during wear process, which corresponds to the wear rate of LDT wheel disc increased gradually. After $3 \times 10^{5}$ cycles, the laser treated zone have been completely removed and the wear rate of LDT wheel disc is approximately equal to that of untreated one. However, the laser treated sample exhibits lower wear rate than the untreated sample during $3 \times 10^{5}$ cycles. In addition, as a frictional pair of the LDT wheel disc, wear rate of the rail disc, is in the range of $10-12 \mu \mathrm{g} / \mathrm{m} /$ $\mathrm{mm}^{2}$ during $2 \times 10^{5}$ cycles and after that the average wear rate keeps around $7 \mu \mathrm{g} / \mathrm{m} / \mathrm{mm}^{2}$. This behavior indicated that laser treated wheel disc has not obvious effect on wear rate of rail disc.

To further understand the LDT wear mechanism, the surface and cross-section the worn LDT wheel disc and untreated wheel disc samples were investigated, as shown in Fig. 6. For the LDT wheel disc, the laser treated spots are protuberant out of the worn surface with only a small quantity of flakelet, where the amount and size of these flakelets increase at edge of the laser treated spots, implying the wear being within mild regime. Fig. 7 is the longitudinal cross-section of the untreated wheel disc taken from $2 \times 10^{5}$ cycles of wear test. As shown in Fig. 7, there is a plastic deformation layer with depth of $110-130 \mu \mathrm{m}$ in cross-section of

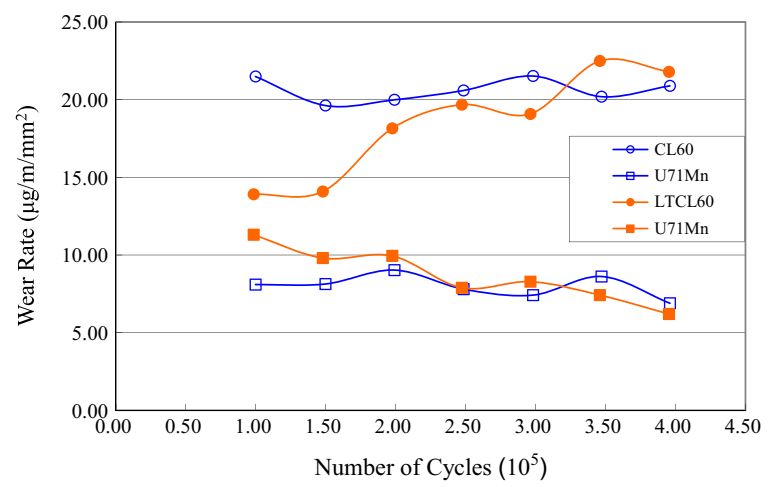

Fig. 5. Wear rates of LDT wheel disc against rail disc and untreated wheel against rail disc. CL60: untreated wheel disc, U71Mn: rail disc, LDTCL60: laser treated wheel disc. 


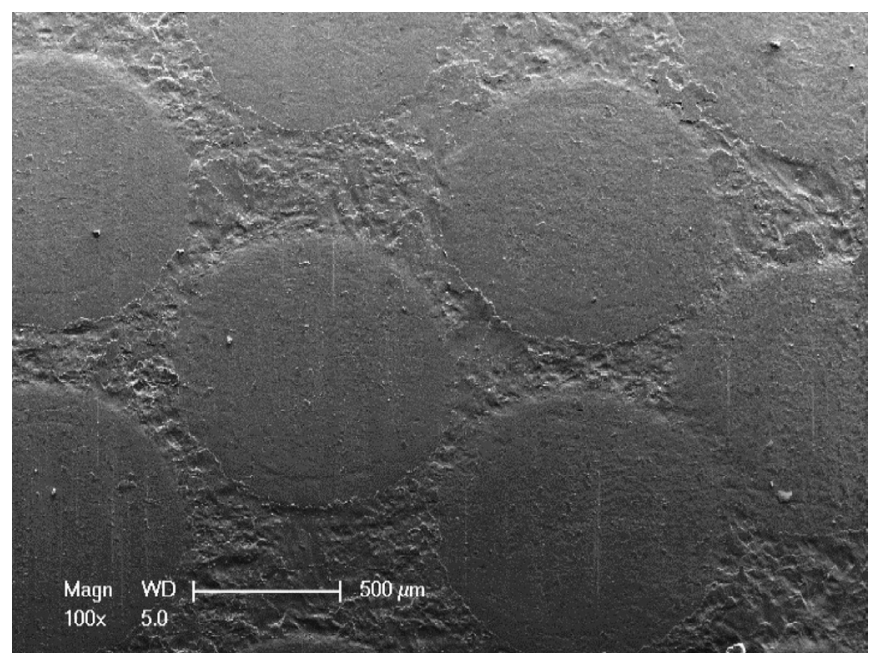

Fig. 6. Morphology of LDT wheel disc after $2 \times 10^{5}$ cycles of wear test.

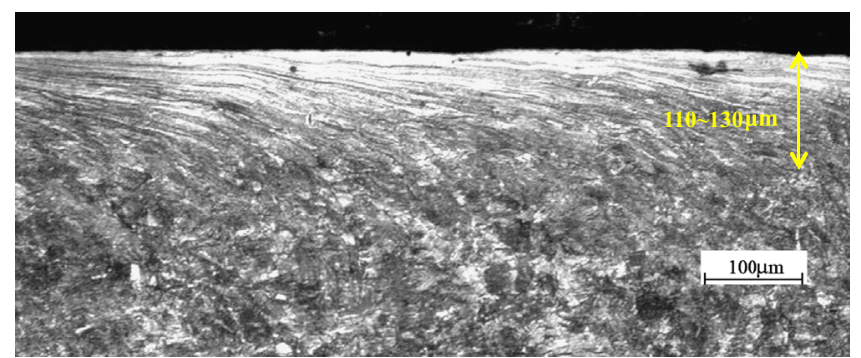

Fig. 7. Longitudinal cross-section of untreated wheel specimen after $2 \times 10^{5}$ cycles of wear test.

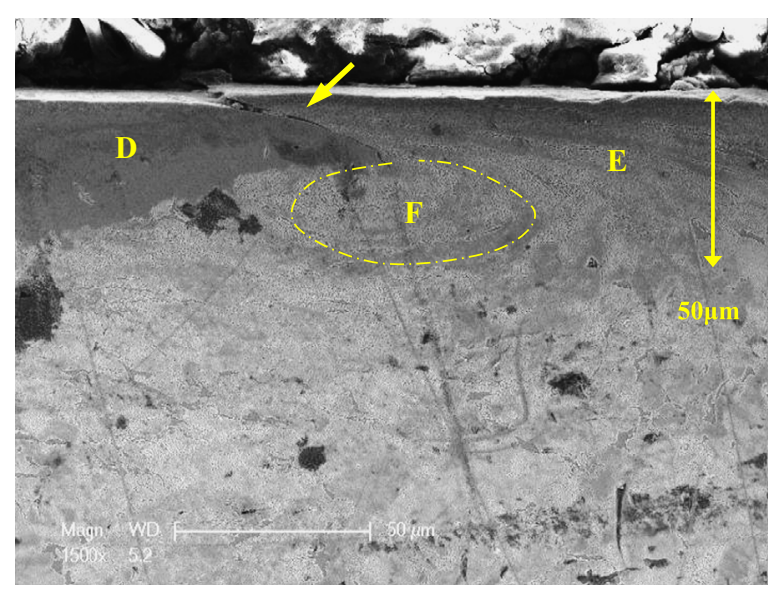

Fig. 8. Longitudinal cross-section of LDT wheel specimen taken from $2 \times 10^{5}$ cycles of wear test. D: LHZ; E: Burble like zone; F: Flowing of pearlite.

worn region for the untreated wheel disc sample and the plastic flow of this layer extends in the direction of tractive force. By contrast, the depth of plastic deformation layer taken from two adjacent laser treated zones is only around $50 \mu \mathrm{m}$ under the same experimental conditions (Fig. 8). This is due to the existence of harder LDT zone that impedes plastic deformation of the region between two adjacent laser treated zones. Furthermore, as shown in Fig. 8, there is a burble like zone marked $\mathrm{F}$ between two adjacent laser treated zones, which provides an evidence for the plastic deformation of pearlite being retarding by hard zone D. The bending of tip of zone $\mathrm{D}$ without cracking indicated that laser treated zone exhibits ductile to a certain extent because of its very fine martensite formed. The stress concentration is primary distributed between zone $\mathrm{D}$ and zone $\mathrm{E}$, as indicated by the presence of crack marked by white arrow in Fig. 8 presents.

\section{Discussion}

To explore the wear mechanism of LDT wheel disc, it is important to analyze the microstructure and mechanical behavior of laser treated zone and its distribution. For a single laser treated zone, its microstructure composed of martensite and retained austenite possesses a higher wear resistance stemming from its higher hardness compared with the untreated one. Shariff et al. claimed that in terms of mass loss, the improvement in wear resistance was more than twofold in laser hardened/melted rail steel as compared to untreated pearlitic substrate [21]. In addition, the higher hardness is also originated from the solid solution strengthening of austenite, high density dislocations and refining grain size [22]. Under condition of rolling-sliding wear, beneficial effect of retained austenite on wear resistance can be discussed as follows: (1) the strain induced phase transformation of austenite into martensite. Colaço et al. claimed that strain induced transformation not only increases the effective hardness of the surface, but also dissipates energy, delaying the fracture process that causes material loss [23]. Young and Bhadeshia also reported that during deformation, the strength of the ductile phase is enhanced via plastic constraint by the surrounding stronger hard phase [28]. This is very similar to structure found in this study that softer LMZ is surrounded by relatively harder LHZ; (2) the retained austenite is helpful to enhance hindering capability for crack propagation $[29,30]$. Lin et al. confirmed that retained austenite effectively decreased or at least retarded microcracking at the surface subject to sliding wear [31]. Zum Gahr summarized that an enhanced amount of austenite and smaller prior austenite grains can improve resistance to rolling contact fatigue and decrease pitting, by increasing work hardening and reducing the rate of crack propagation [24]. Although the references did not clearly state what the critical value is, it implied that a positive effect on rolling-sliding wear resistance with around 10\% retained austenite. In this study it is deduced the amount of retained austenite in LDT zone is no more than $10 \%$, the positive effect of austenite on improving wear resistance might be concluded; (3) when the subsurface deformation occurred, cracks are nucleated below the surface, rather than near the surface resulting from the triaxial compressive stress that exists just below the contact regions. As reported by James, compressive residual stress was existed on laser treated zone of medium carbon steel if there is no additional reheat treatment [19]. Thus LDT zones with its original compressive stress act as an individual hardening point resist local plastic deformation and retard crack nucleation and propagation.

Furthermore, the untreated area between laser treated zones is also influenced by the discrete distribution of laser treated zones. As stated above the depth reduction of plastic deformation between untreated zone results from the distribution of laser treated zone. The less depth means higher resistance to plastic deformation and thus higher wear resistance.

\section{Conclusions}

The study demonstrated the feasibility of improving rolling and sliding wear resistance in wheel steel by laser dispersed treatment. By carrying out two rollers rolling and sliding wear test with slip ratio around $0.5 \%$ and traction coefficient 0.263 , and analysis the 
microstructure and mechanical behavior of individual laser treated zone and its distribution, results are the followings:

1. The processing of laser dispersed treatment, avoiding the disadvantages of multiple passes by overlapping laser tracks, improve wear resistance of wheel steel disc in rolling-sliding condition controlled by individual harder laser zone and its distribution.

2. The higher wear resistance of laser treated wheel is attributed to its microstructure and stress status within laser treated zone.

3. The laser dispersed treatment on wheel may be a useful technique to improve the wear resistance of wheel without obvious negative effect on wear rate of rail.

\section{Acknowledgements}

The work reported in this paper was supported by Ministry of Science and Technology of the PR China (Grant No. 2009BAG12A01B10-2). Part of the financial support from the Chinese Academy of Sciences (Grant No. KJCX2-EW-L03) is acknowledged.

\section{References}

[1] M.L. Ki, A.P. Andreas, Wear of conventional pearlitic and improved bainitic rail steels, Wear 259 (1-6) (2005) 391-399.

[2] F.C.R. Hernandez, N.G. Demas, D.D. Davis, Mechanical properties and wear performance of premium rail steels, Wear 263 (2007) 766-772.

[3] M.R. Zhang, H.C. Go, Microstructure and mechanical properties of railway wheels manufactured with low-medium carbon Si-Mn-Mo-V steel, J. Univ. Sci. Technol. Beijing 15 (2) (2008) 125-131.

[4] R.O. Olivares, C.I. Garcia, A. DeArdo, Advanced metallurgical alloy design and thermomechanical processing for rails steels for North American heavy haul use, Wear 271 (2011) 364-373.

[5] M.R. Zhang, H.C. Gu, Fracture toughness of nanostructured railway wheels, Eng. Fract. Mech. 75 (18) (2008) 5113-5121.

[6] W.M. Steen, J. Mazumder, Laser Material Processing, fourth ed., SpringerVerlag, London, 2010.

[7] R.J. DiMelfi, P.G. Sanders, B. Hunter, et al., Mitigation of subsurface crack propagation in railroad rails by laser surface modification, Surf. Coat. Technol. 106 (1) (1998) 30-43.

[8] S. Aldajah, O.O. Ajayi, G.R. Fenske, et al., Investigation of top of rail lubrication and laser glazing for improved railroad energy efficiency, J. Tribol. Trans. ASME 125 (3) (2003) 643-648.

[9] E.J.M. Hiensch, F.J. Franklin, J.C.O. Nielsen, Prevention of RCF damage in curved track through development of the INFRA-STAR two-material rail, Fatigue Fract. Eng. Mater. Struct. 26 (10) (2003) 1007-1017.
[10] S. Niederhauser, B. Karlsson, Fatigue behaviour of $\mathrm{Co}-\mathrm{Cr}$ laser cladded stee plates for railway applications, Wear 258 (7-8) (2005) 1156-1164.

[11] M.S.F. Lima, H. Goldenstein, Structure of laser remelted surface of cast irons, Surf. Eng. 16 (2) (2000) 127-130.

[12] H.M. Wang, H.W. Bergmann, Rapid graphitization of a pulsed laser remelted ductile cast iron during multipass overlap melting, Metall. Mater. Trans. A 26 (4) (1995) 793-800.

[13] Y. Iino, K. Shimoda, Effect of overlap pass tempering on hardness and fatigue behavior in laser heat-treatment of carbon-steel, J. Mater. Sci. Lett. 6 (10) (1987) 1193-1194.

[14] B.A. Van Brussel, H.J. Hegge, J.Th. De Hosson, et al., Development of residual stress and surface cracks in laser treated low carbon steel, Scr. Metall. Mater. 25 (1991) 779-784.

[15] B.A. Van Brussel, J.Th. De Hosson, Residual stresses in the surface layer of lasertreated steels, Mater. Sci. Eng., A 161 (1993) 83-89.

[16] J.R. Jiang, L.J. Xue, S.D. Wang, Discrete laser spot transformation hardening of AISI 01 tool steel using pulsed Nd:YAG laser, Surf. Coat. Technol. 205 (21-22) (2011) 5156-5164.

[17] Z.Y. Li, H.Y. Zhao, Y. Gu, et al., Fatigue crack propagation in laser alloyed ductile cast iron surface, J. Laser Appl. 25 (2013) 012003.

[18] C. Papaphilippou, M. Jeandin, Spot laser hardening, J. Mater. Sci. Lett. 15 (12) (1996) 1064-1066.

[19] M.R. James, D.S. Gnanamuthu, R.J. Moores, Mechanical state of laser melted surfaces, Scr. Metall. 18 (1984) 357-361.

[20] C.Y. Cui, J.D. Hu, Y.H. Liu, et al., Formation of nano-crystalline and amorphous phases on the surface of stainless steel by Nd:YAG pulsed laser irradiation, Appl. Surf. Sci. 254 (21) (2008) 6779-6782.

[21] S.M. Shariff, T.K. Pal, G. Padmanabham, et al., Sliding wear behavior of lase surface modified pearlitic rail steel, Surf. Eng. 26 (3) (2010) 199-208.

[22] R. Vilar, R. Colaco, A. Almeida, Laser surface treatment of tool steels, Opt Quantum Electron. 27 (1995) 1273-1289.

[23] R. Colaço, C. Pina, R. Vilar, Influence of the processing conditions on the abrasive wear behaviour of a laser surface melted tool steel, Scr. Mater. 41 (7) (1999) 715-721.

[24] K.H. Zum Gahr, Microstructure and Wear of Materials, Elsevier, Amsterdam, 1987.

[25] R. Lewis, U. Olofsson, Mapping rail wear regimes and transitions, Wear 257 (7-8 (2004) 721-729.

[26] R. Lewis, R.S. Dwyer-Joyce, U. Olofsson, et al., Mapping railway wheel material wear mechanisms and transitions, Proc. Inst. Mech. Eng. Part F J. Rail Rapid Transit 224 (F3) (2010) 125-137.

[27] A. Ekberg, E. Kabo, Fatigue of railway wheels and rails under rolling contact and thermal loading-an overview, Wear 258 (2005) 1288-1300.

[28] C.H. Young, H.K.D.H. Bhadeshia, Strength of mixtures of bainite and martensite, Mater. Sci. Technol. 10 (3) (1994) 209-214.

[29] P.F. Stratton, Optimising nano-carbide precipitation in tool steels, Mater. Sci Eng., A 449-451 (2007) 809-812.

[30] Y. Wang, T.Q. Lei, J.J. Liu, Tribo-metallographic behavior of high carbon steels in dry sliding III. Dynamic microstructural changes and wear, Wear 231 (1) (1999) 20-37.

[31] P.Y. Lin, Y.F. Zhu, H. Zhou, et al., Wear resistance of a bearing steel processed by laser surface remelting cooled by water, Scr. Mater. 63 (8) (2010) 839-842. 